\title{
An approach to endomyocardial biopsy interpretation
}

\author{
K S Cunningham, J P Veinot, J Butany
}

J Clin Pathol 2006;59:121-129. doi: 10.1136/icp.2005.026443

The endomyocardial biopsy (EMB) remains the gold standard mode of investigation for diagnosing many primary and secondary cardiac conditions. Through a percutaneous and transvenous route, tissue fragments are generally procured from the right ventricular septum, with very few complications. Widespread use of EMB followed the development of heart transplantation as a means to follow allograft rejection. It has since been useful in helping to diagnose conditions affecting the heart, including cardiomyopathies, myocarditis, infiltrative lesions, arrhythmias, and drug toxicities. The procedure has also been used as a research tool to investigate the natural history of disease and the cardiotoxicity of new medications. This review presents an approach to the evaluation of the $E M B$, which is particularly directed towards those who may be asked to interpret such biopsies, but are not dedicated cardiovascular pathologists. Through a systematic evaluation of the endocardium, myocardium, interstitium, and intramural vessels, in the context of a complete clinical history, enough information can be deduced to diagnose or exclude specific conditions of clinical value.

See end of article for authors' affiliations .....................

Correspondence to: Dr J Butany, Department of Pathology E4-301, Toronto General Hospital, Toronto, Ontario M5G 2CA

Canada; jagdish.butany@ uhn.on.ca

Accepted for publication 14 July 2005
$\mathrm{T}$ he endomyocardial biopsy (EMB) was established as a diagnostic interventional procedure after the development and application of cardiac catheterisation and cardiothoracic surgery. Although necropsy had already provided much of the histopathological characterisation of primary or secondary myocardial disease, successful identification and treatment of such conditions had to await further technological developments. The 1950s introduced the open surgical biopsy of the heart, followed by needle biopsies using the modified Vim-Silverman needle through a limited thoracotomy or transthoracic approach. ${ }^{12}$ However, the occurrence of pneumothoraces and cardiac tamponade limited its acceptance. Sakakibara and Konno introduced the biopsy catheter or "bioptome" in 1962 as a means of sampling endomyocardium, in contrast to previous epicardial and transmural sampling. ${ }^{3}$ The benefit of this new biopsy catheter was adequate endomyocardial sampling, with fewer complications, which occurred via a transvascular approach under fluoroscopic guidance. Access through the neck veins precluded the necessity for "cut downs" for vascular exposure.
Further modifications have been made in catheter design, such as sheathing and increasing flexibility and manoeuvrability, in addition to the development of the left ventricular endomyocardial biopsy. ${ }^{4-6}$ The EMB found limited use worldwide until advances in cardiac transplantation necessitated a means to monitor graft rejection. In 1972, the Stanford group developed a new percutaneous, flexible biopsy forceps that could serially obtain right ventricular endomyocardial biopsies after transplantation. ${ }^{7}$ With this new tool, the right ventricular heart biopsy procedure gained acceptance as a clinically useful interventional technique, and the use of cardiac biopsies to investigate other non-transplant related pathologies became more commonplace.

\begin{abstract}
"The endomyocardial biopsy found limited use worldwide until advances in cardiac transplantation necessitated a means to monitor graft rejection"
\end{abstract}

\section{CLINICAL INDICATIONS}

Serially acquired endomyocardial specimens to monitor graft rejection are today the most frequent indication for endomyocardial biopsy. However, EMB continues to be useful for the investigation of infiltrative disorders of the myocardium, primary cardiomyopathies, myocarditis, endocardial fibrosis (as a way to help distinguish between constrictive and restrictive pathology), drug toxicity, arrhythmias, and neoplasia (figs 1, 2). ${ }^{8-14}$ Although there are entities such as cardiac amyloidosis and myocarditis that can be definitively diagnosed, many disease states, such as dilated cardiomyopathy (DCM; which may be seen as a terminal structural and functional manifestation of several conditions affecting the heart, both primarily and secondarily), show non-specific histopathological features. In particular, it should be emphasised that dilated cardiomyopathy is not a histological diagnosis per se, but requires significant clinical-pathological correlation, which is essential for cardiac biopsy interpretation, because a definitive diagnosis is often not possible on the basis of morphology alone. The major role of the biopsy in this setting is to enable the pathologist to rule out various conditions provided by the differential diagnosis. An example includes the aforementioned differentiation between constrictive pericarditis and

Abbreviations: DCM, dilated cardiomyopathy; EMB, endomyocardial biopsy; TEM, transmission electron microscopy 

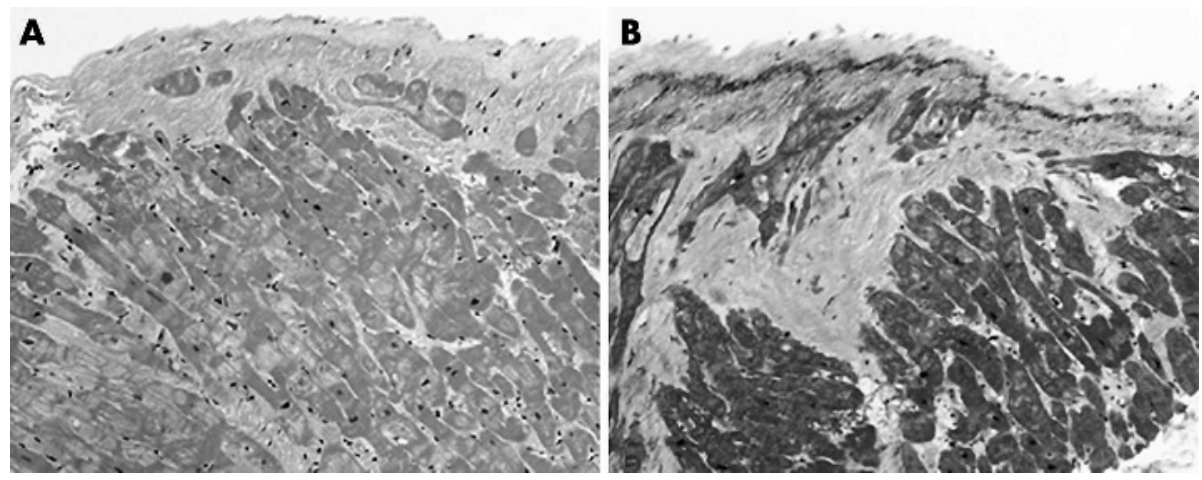

Figure 1 (A) Biopsy from a 45 year old woman with dilated cardiomyopathy. Note the endocardial fibrosis, myocyte hypertrophy, myocyte nuclei, and moderate interstitial fibrosis (haematoxylin and eosin staining; original magnification, $\times 10$ ). (B) Elastic trichrome stain of the same case showing thickened fibrous endocardium with considerable interstitial fibrosis (original magnification, $\times 10$ ).

restrictive cardiomyopathy or infiltrative myocardial disease. The clinical differentiation of myocarditis from DCM of a different aetiology is often difficult if not impossible. Although DCM and myocarditis may coexist, another instance where the EMB is of value to clinicians is in differentiating between established DCM with no evidence of myocardial inflammation and active myocarditis. The EMB is also very useful in the evaluation of drug toxicity, including the investigation of potentially cardiotoxic agents, such as the chemotherapeutic agents Herceptin (Genentech Inc, San Francisco, California, USA) and doxorubicin. ${ }^{12}{ }^{15}$ Finally, there are less conventional but documented uses of EMB, such as the investigation of the cardiovascular sequelae of anabolic steroids, thalassaemic heart disease, and human immunodeficiency virus in myocardial disease (table 1). ${ }^{16-18}$

"Although dilated cardiomyopathy and myocarditis may coexist, another instance where the endomyocardial biopsy is of value to clinicians is in differentiating between established dilated cardiomyopathy with no evidence of myocardial inflammation and active myocarditis"

\section{TECHNICAL CONSIDERATIONS}

The easily assessable anatomy of venous return to the heart with peripheral access makes the right ventricle an attractive location for tissue sampling. The interventricular septum is the preferred biopsy site because of its thickness, compared with the right ventricular free wall, its continuity with the left ventricle, and its location in the natural path of blood flow facilitating vascular access. The drawback is that repeated EMB sampling results in a restricted region of endocardium being assessed and may result in interpretive error if the clinician is not aware of this point. The likelihood of free wall perforation and haemopericardium/tamponade is
Table 1 Clinical indications for endomyocardial biopsy

Endomyocardial biopsy should be used to assess the following conditions

(1) Allograft heart rejection surveillance

(2) Myocarditis

(3) Amyloidosis

(4) Haemochromatosis

(5) Sarcoidosis

(6) Fabry's disease

(7) Loeffler's endomyocarditis

(8) Mastocytosis

(9) Neoplasia (benign, primary malignant, or metastatic)

(10) Cardiomyopathies (hypertrophic, dilated, or restrictive)

(11) Drug toxicity (for example, Adriamycin, Herceptin)

(12) Arrhythmia

(13) Unexplained chest pain

(14) Unexplained congestive heart failure

(15) Differentiation between restriction and pericardial constriction

a major reason for consciously avoiding the free wall for biopsy. Fluoroscopy is the standard imaging modality used to guide the biopsy catheter, although echocardiography is now used by some groups, with claims of superior visualisation of heart anatomy and earlier recognition of acute procedural complications. ${ }^{19}$

The right ventricle has a thinner wall in the region of the apex adjacent to the interventricular septum. This is important, because this is the site where perforation could occur with relative ease, especially in those who have appreciable fatty replacement of the right ventricular free wall. The site of tissue sampling is also pertinent if one considers cardiomyocyte disarray as potential evidence of cardiomyopathy, in particular hypertrophic cardiomyopathy. Myocyte disarray is normally encountered in the region of the
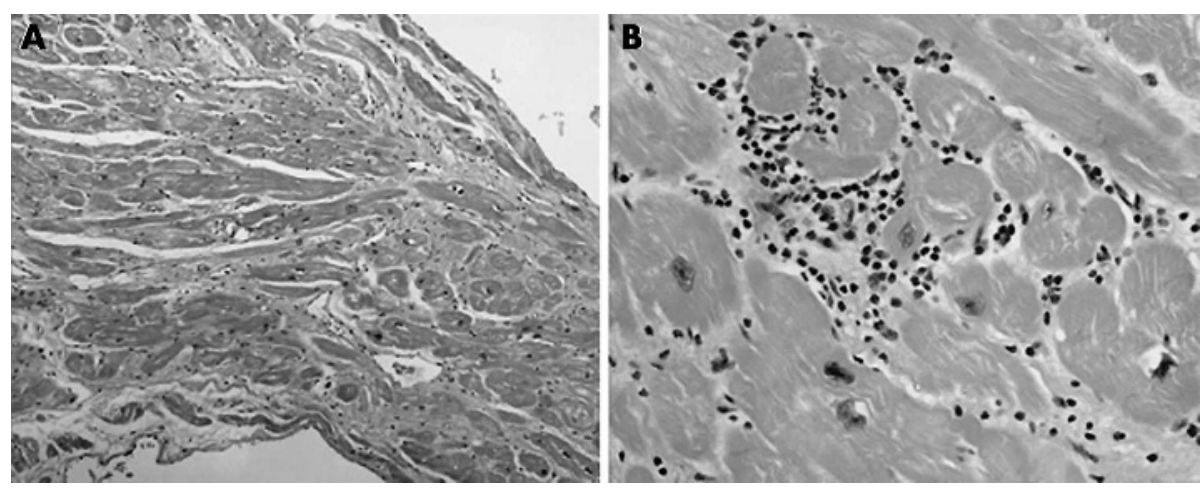

Figure 2 (A) Biopsy from a 37 year old man who presented with acute onset, congestive heart failure. The biopsy shows active myocarditis with morphological changes consistent with dilated cardiomyopathy (haematoxylin and eosin staining; original

magnification, $\times 10$ ). (B) A section from the same patient demonstrating foci of lymphocyte infiltration and active myocyte necrosis (haematoxylin and eosin staining; original magnification, $\times 20$ ). 
Table 2 Special stains for endomyocardial biopsies

\begin{tabular}{|c|c|}
\hline Stain & Frequent indication \\
\hline Methyl green pyronine & $\begin{array}{l}\text { Lymphocytes (myocarditis/allograft } \\
\text { rejection) }\end{array}$ \\
\hline Movat pentachrome & Connective tissue/vasculature \\
\hline Masson elastic trichrome & Connective tissue/vasculature \\
\hline Prussian blue & Iron \\
\hline Sulfated Alcian blue & Iron and amyloid \\
\hline Congo red & Amyloid \\
\hline Ziehl-Neelsen & Granulomas (acid fast bacilli) \\
\hline Periodic acid Schiff & $\begin{array}{l}\text { Intramyocardial glycogen/vasculature/ } \\
\text { fungi }\end{array}$ \\
\hline Gram & Bacteria (endocarditis) \\
\hline Gomori methenamine silver & Fungi \\
\hline $\begin{array}{l}\text { Haematoxylin phloxine } \\
\text { saffron }\end{array}$ & Connective tissue \\
\hline Methyl violet & Amyloid \\
\hline
\end{tabular}

ventricular apex and at the junction of the ventricular free wall with the interventricular septum, and should not be interpreted as evidence of primary myocardial disease.

Although infrequently performed, left ventricular biopsies are technically possible and may provide clinically useful information in diagnosing conditions affecting principally the left ventricular wall(s). These biopsies were generally performed by needle biopsy at the time of open heart surgery or via a trans-septal approach; however, the procedure may be performed percutaneously through the femoral artery, retrograde through the aortic valve into the left ventricle. ${ }^{20}$ There are few indications aside from those processes resulting in left ventricular specific pathology, such as left ventricular masses, that require left ventricular EMB for diagnosis. The arteriotomy requires systemic heparinisation to prevent peripheral embolisation during the procedure. Systemic or cerebral arterial embolisation (atheroembolic or thromboembolic) may have devastating clinical consequences, so that many cardiologists perform right ventricular biopsies preferentially. Atrial biopsies may also be taken, although this is exceedingly rare. ${ }^{21}$

The endomyocardial biopsy is generally an outpatient procedure. The tissue sample procured is usually a $1-2 \mathrm{~mm}$ cube of endocardium and myocardium. After extraction of the tissue fragment the cardiologist should be careful not to

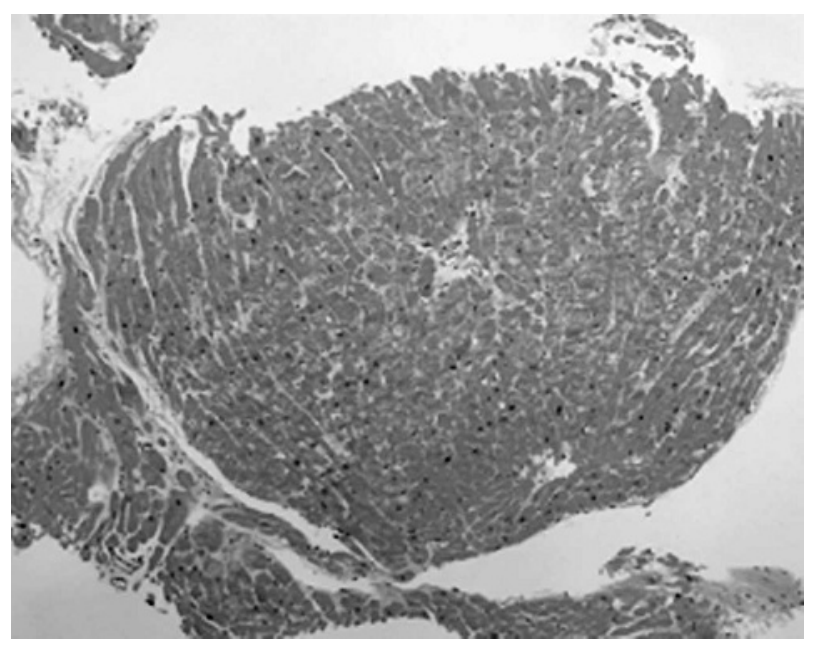

Figure 3 A normal endomyocardial biopsy from a 58 year old man as part of allograft transplant surveillance (grade 0). For an adequate biopsy, a minimum of four fragments with less than $50 \%$ being noninterpretable tissue (table 6) is recommended (haematoxylin and eosin staining; original magnification, $\times 2.5$ ). remove the specimen with forceps, but rather to "move it" gently with a needle off the biopsy catheter and place it either on to a piece of saline soaked gauze or directly into $10 \%$ neutral buffered formalin. The fixative should be at room temperature to prevent additional contraction band artefact. Histological preparation, embedding, staining (see below), reading, and reporting of the diagnosis within 24 hours is standard. Faster processing - within a few hours-is possible, if indicated; however, this service rarely results in immediate clinical intervention. Frozen sections are usually not indicated and may make interpretation difficult. For diagnostic EMB, a sample should always be set aside for transmission electron microscopy (TEM). In biopsies obtained to investigate neoplasia or infiltrative disease, a specimen should also be frozen for additional molecular studies, immunofluorescence, or immunohistochemistry that may be required for tumour typing or amyloid classification.

\section{"The easily assessable anatomy of venous return to the heart with peripheral access makes the right ventricle an attractive location for tissue sampling"}

Standard histological preparation requires paraffin wax embedding, followed by ribbons of $4 \mu \mathrm{m}$ thick sections mounted on glass slides. In Toronto, 15 such slides are obtained, representing nearly the entire thickness of the block of tissue. Slides are numbered sequentially, with slide numbers 1, 3, 5, 9, 11, and 15 being stained with haematoxylin and eosin for histomorphological characterisation, and slides 7 and 13 being stained with the elastic trichrome technique to visualise collagenous and elastic tissue. The remaining sections are kept for special stains if necessary. In Ottawa, the whole block is sectioned with intervening sections retained for special purposes. The entire block is cut through to avoid missing myocarditis. An iron stain is done on all men and on all postmenopausal women, regardless of the indication, so as not to miss an iron storage disorder, a potentially treatable condition. Congo red staining is performed on all patients over the age of 50 years (thick $10-15 \mu \mathrm{m}$ sections are recommended). The Masson elastic trichrome or Movat pentachrome stains are particularly useful to assess interstitial fibrosis, cardiomyocyte necrosis, and disarray, in addition to the presence of contraction bands. Other options for staining sections are readily available (table 2) and may be considered where indicated.

Evaluation of sample adequacy for the International Society of Heart and Lung Transplantation grading scheme requires a minimum of four good endomyocardial tissue fragments, with less than $50 \%$ of each fragment being fibrous tissue, thrombus, or other non-interpretable tissues (such as crush artefact or poorly processed fragments) (figs 3,4). ${ }^{22}$ The sensitivity of detecting transplant rejection can approach $98 \%$ with five adequate biopsy fragments, yet more than six samples does not appear to increase diagnostic yield. ${ }^{23}{ }^{24}$ More fragments may be necessary for focal myocardial diseases, such as myocarditis, and as many as 10 pieces may be needed for confident interpretation. The greatest potential limitation to EMB interpretation is sampling error. The adequacy of tissue fragments is very important for correct diagnostic accuracy and interpretation.

Special studies such as TEM are indicated for assessing conditions suggestive of cardiomyopathy, infiltrative disorders (such as amyloidosis or glycogen storage disorders), viral myocarditis, and possibly drug toxicity (table 3$).{ }^{23}$ Universal fixative is recommended for TEM preparation, although any $2 \%$ glutaraldehyde based fixative will suffice. ${ }^{23}$ Fresh or flash frozen tissue is not ideal for the standard preparation of tissue fragments because of the potential for ice crystal 

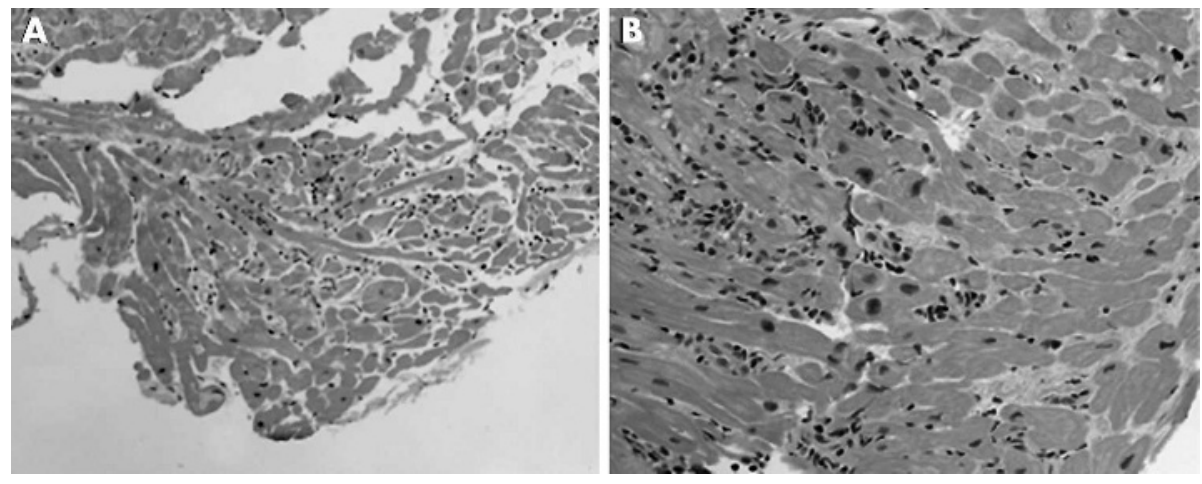

Figure 4 (A) Biopsy from a 61 year old man showing grade 1A rejection with foci of lymphocytic infiltrates and no evidence of myocyte damage (haematoxylin and eosin staining; original magnification, $\times 5.0)$. (B) Grade 3A rejection showing significant lymphoid infiltration and myocyte necrosis. This figure as shown would represent grade $3 \mathrm{~A}$ graft rejection; however, this biopsy sample consisted of diffuse lymphocytic infiltrates and myocyte necrosis in all of the biopsy pieces and the sections examined, and was diagnosed as grade $3 \mathrm{~B}$ rejection (haematoxylin and eosin staining; original magnification, $\times 20$ ).

artefacts. However, such samples are suitable for immunofluorescence, culture, polymerase chain reaction or reverse transcriptase PCR polymerase chain reaction based investigations-for example, as a means of identifying virus to support a diagnosis of viral myocarditis. ${ }^{25}$

The usefulness of immunohistochemistry is dependent on the information sought. Amyloid may be immunophenotyped, providing important prognostic information, and the results may suggest that additional investigations should be performed to evaluate plasma cell dyscrasia. ${ }^{26}$ Immunophenotyping of lymphocytes in acute cellular allograft rejection, myocarditis, or lymphoma can be diagnostically useful. ${ }^{27} 28$ Immunohistochemistry and immunofluorescence are also used to assess acute vascular rejection or biopsy negative cardiac transplant rejection. ${ }^{29}{ }^{30}$ Immune related cardiomyopathy may also be considered with use of appropriate immunostains.

Clinically relevant complications from EMB sampling are rare, and are estimated at $1-2 \%$, with death an exceptionally rare event at less than $0.2 \%$ (table 4 ). ${ }^{22}{ }^{31}$ The most important potential "complication" of EMB is sampling error-missing pathology that is focal in nature or potentially limited to the left ventricle. Cardiologists attempt to compensate for this possibility by obtaining multiple tissue fragments; however, great importance must be given to the clinico-pathological interpretation of the disease entity at hand to arrive at the final diagnosis.

"Clinically relevant complications from endomyocardial biopsy sampling are rare, and are estimated at $1-2 \%$, with death an exceptionally rare event at less than $0.2 \%{ }^{\prime \prime}$

Although not always a practical consideration, the value of consultation with a dedicated cardiovascular pathologist cannot be overstated. Surgical pathologists within an academic health centre may routinely see a sufficient number of allograft biopsies to be comfortable with their diagnostic challenges. However, biopsies to assess other conditions such

Table 3 Adjunctive studies available for endomyocardial biopsy material

Adjunctive studies

(1) Polymerase chain reaction (viral genomes-myocarditis)

(2) Transmission electron microscopy (myocarditis/cardiomyopathy/ amyloidosis)

(3) Immunohistochemistry (acute allograft rejection/amyloidosis/ neoplasm/cardiomyopathy)

(4) Histochemical stains (storage disease/metabolic disease)

(5) Immunofluorescence (rejection/cardiomyopathy) as drug toxicity, infiltrative disorders, myocarditis, and specimens supporting the diagnosis of DCM are uncommon and should ultimately be evaluated at a centralised facility that serves a large referral base. Dedicated cardiovascular pathologists are familiar with primary and secondary myocardial disease and the common artefacts associated with sampling itself, in addition to the histomorphological changes associated with prosthetic device implantation (pacemaker leads/ventricular assist devices) that may be present in the failing heart.

\section{FINDINGS AND IMPORTANCE}

The approach to an endomyocardial biopsy specimen involves the examination of multiple serial sections to assess histomorphology and to "reconstruct" identified pathologies within the sample. Similar to other medical tissue biopsy specimens, careful evaluation of each component of the biopsy specimen including endocardium, myocardium, interstitium, and vasculature is crucial to avoid missing diagnostic entities (table 5). The final report should include the biopsy site, type of biopsy, and clinically relevant information including diagnosis, grading scheme, and a detailed microscopic description that may not be useful to the clinician, but may facilitate future research endeavours. Finally, the interpretation of cardiac biopsies should never be performed in the absence of appropriate and adequate clinical information that may help to rationalise non-specific findings, direct further investigations, and allow definitive diagnoses (or

Table 4 Complications of endomyocardial biopsies

\begin{tabular}{l}
\hline Complications \\
\hline Major complications \\
Haemopericardium \\
Tamponade \\
Mediastinitis \\
Pneumothorax \\
Air embolism \\
Pneumopericardium \\
Thromboembolism \\
Myocardial infarction \\
Transmission of infectious organism (such as hepatitis B/C) \\
Tricuspid or mitral valve damage with possible severe regurgitation \\
Pericardial fibrosis/thickening \\
Minor complications \\
Chest pain (transient) \\
ECG abnormalities (transient) \\
Nerve palsy \\
Rupture of chordae tendineae \\
Tricuspid valve damage with regurgitation \\
Haematoma \\
Hypotension \\
Deep vein thrombosis \\
Vascular fistulae \\
\hline ECG, electrocardiogram.
\end{tabular}




\begin{tabular}{|c|c|}
\hline Tissue/Histopathology & Consider in diagnosis \\
\hline \multicolumn{2}{|l|}{ Endocardium } \\
\hline \multirow[t]{11}{*}{ Fibrosis } & Organised thrombus \\
\hline & Healed biopsy site \\
\hline & Myocardial infarct \\
\hline & Healed acute rejection site \\
\hline & Graft procurement injury \\
\hline & Adjacent prosthetic device \\
\hline & $\begin{array}{l}\text { Abnormal blood flow haemodynamics } \\
\text { Hypereosinophilic syndrome }\end{array}$ \\
\hline & $\begin{array}{l}\text { Hypereosinophilic syndrome } \\
\text { Mastocytosis }\end{array}$ \\
\hline & Endocardial fibroelastosis \\
\hline & Healed myocarditis \\
\hline & $\begin{array}{l}\text { Cardiomyopathy } \\
\text { Drug toxicity }\end{array}$ \\
\hline Ulceration/necrosis & Adjacent prosthetic device \\
\hline & Healing biopsy site \\
\hline & Hypereosinophilic syndrome \\
\hline \multicolumn{2}{|l|}{ Myocardium } \\
\hline \multirow{4}{*}{ Hypertrophy } & Hypertrophic/dilated cardiomyopathy \\
\hline & $\begin{array}{l}\text { Pressure/volume overloaded ventricle } \\
\text { Storage disorders }\end{array}$ \\
\hline & Primary myopathies (Duchenne's/Becker's) \\
\hline & Muscular dystrophies (myotonic) \\
\hline \multirow[t]{5}{*}{ Fibre disarray } & Hypertrophic cardiomyopathy \\
\hline & Healed biopsy site \\
\hline & Ventricular apex \\
\hline & Junction of free wall and interventricular \\
\hline & \\
\hline \multicolumn{2}{|l|}{ Interstitium } \\
\hline \multirow[t]{8}{*}{ Fibrosis } & Healed biopsy site \\
\hline & Myocardial infarct \\
\hline & Healed acute rejection site \\
\hline & $\begin{array}{l}\text { Adjacent prosthetic device } \\
\text { Hypereosinophilic syndrome }\end{array}$ \\
\hline & Mastocyłosis \\
\hline & Healed myocarditis \\
\hline & Cardiomyopathy \\
\hline & Drug toxicity \\
\hline \multirow[t]{5}{*}{ Lymphocytes } & Allograft rejection (focal or diffuse) \\
\hline & Quilty lesion (A or B) \\
\hline & Normal allograft (sparse and diffuse) \\
\hline & $\begin{array}{l}\text { Myocarditis/dilated cardiomyopathy } \\
\text { Post transplant lymphoproliferative disease } \\
\text { Lymphoma }\end{array}$ \\
\hline & Leukaemia \\
\hline \multirow[t]{3}{*}{ Neutrophils } & $\begin{array}{l}\text { Bacterial/fungal endocarditis ( } \pm \text { necrosis) } \\
\text { Fulminant acute allograft rejection }\end{array}$ \\
\hline & $\begin{array}{l}\text { Fulminant myocarditis } \\
\text { Hypereosinophilic syndrome (+eosinophils) }\end{array}$ \\
\hline & Sepsis \\
\hline \multirow{4}{*}{ Eosinophils } & Hypereosinophilic syndrome (Loeffler's) \\
\hline & Hypersensitivity (for example, drug) \\
\hline & Fulminant allograft rejection \\
\hline & Parasitic infection (Chagas) \\
\hline \multirow{11}{*}{ Intramural vessel changes } & Sarcoidosis \\
\hline & Amyloidosis \\
\hline & Aschoff nodules \\
\hline & Foreign body reaction \\
\hline & Giant cell myocarditis \\
\hline & Allograft vasculopathy \\
\hline & Hypertrophic cardiomyopathy \\
\hline & Systemic arterial hypertension \\
\hline & Amyloidosis \\
\hline & Collagen vascular disorders \\
\hline & Mitral valve prolapse \\
\hline
\end{tabular}

exclusion of diagnoses).

An overview of some of the major histopathological findings in each myocardial biopsy component and their significance is presented below.

\section{Endocardium}

The normal endocardium is composed of an endothelial cell layer, a subendothelial layer of loose collagen and fibroblasts, and an elastic layer, often associated with collagen and smooth muscle cells. These endocardial layers appear at times to be ill defined and can have a variable thickness depending on location. For instance, the right ventricular outflow tract has a thicker endocardial layer than the inflow surface. A separate subendocardial layer between the endocardium and myocardium is composed of thick collagen, elastin, fibroblasts, and smooth muscle cells and may have associated blood vessels, Purkinje fibres, and adipocytes, particularly in the right ventricle. This layer is continuous with the extracellular interstitium surrounding cardiomyocytes. $^{32}$

The most important pathological alteration of the endocardium is thickening, in general as a result of a combination of fibrosis and elastosis. It is possible that this change can be overdiagnosed if the biopsy specimens are tangentially embedded. Serial sections solve this interpretative error. Endocardial fibrosis may be a non-specific finding or representative of the specific pathology occurring. It may be the result of altered blood flow haemodynamics or may be a secondary effect of repeated EMB sampling or catheterisation related endomyocardial injury (healing trauma) (fig 5). The differentiation between these causes requires a careful account of clinical information. For example, in the allograft heart repeated biopsies of the right ventricular septum result in endocardial fibrosis at healed biopsy sites. These may appear in different stages of repair and are often found with myocyte disarray and/or adipocytes (fig 6). ${ }^{31}$ Thrombus can also be seen as a result of a previous biopsy or a primary pathological process, such as Loeffler's endomyocarditis, that has secondarily organised. ${ }^{33}$ Organised mural thrombi may also be found in association with cardiomyopathies, intracardiac masses, or a prosthetic device. Fresh fibrin platelet thrombus may form at the site of the biopsy itself or on the catheter sheath, and should not be over interpreted as an important finding. Subendocardial fibrosis may be the result of a healed myocardial infarct. Other rare entities such as primary endocardial fibroelastosis are known, so that clinical correlation is imperative. ${ }^{34}$

\section{"The most important pathological alteration of the endocardium is thickening, in general as a result of a combination of fibrosis and elastosis"}

Various cellular infiltrates can be seen in the endocardium. In addition to the fibroblastic/smooth muscle cell proliferations associated with organisation and fibrosis, the most common focal endocardial cellular infiltrate seen at endomyocardial biopsy is secondary to the "Quilty" effect. ${ }^{35}$ Quilty lesions are endocardial inflammatory infiltrates associated with allograft heart transplantation coupled with cyclosporine A immunomodulation. Interestingly, Quilty lesions are not seen in native endocardium in patients undergoing similar treatment to prevent graft rejection of other organs. ${ }^{36}$ Quilty A lesions are confined to the endocardium and are composed primarily of small lymphocytes, with occasional small capillaries, macrophages, and plasma cells. Quilty B lesions are similar, but they extend into the underlying subendocardium and myocardial interstitium, surrounding cardiomyocytes that may or may not show focal myocyte necrosis. Quilty lesions (A or B) do not appear to be clinically relevant.

When assessing focal myocardial inflammatory cell infiltrates near but apparently not involving the endocardial surface, it is important to evaluate deeper sections within the tissue block to determine whether the lesion becomes continuous with the overlying endocardium, and is in fact a Quilty lesion. Endocardial infiltration by neoplastic cells from a metastatic process is possible but uncommon. 

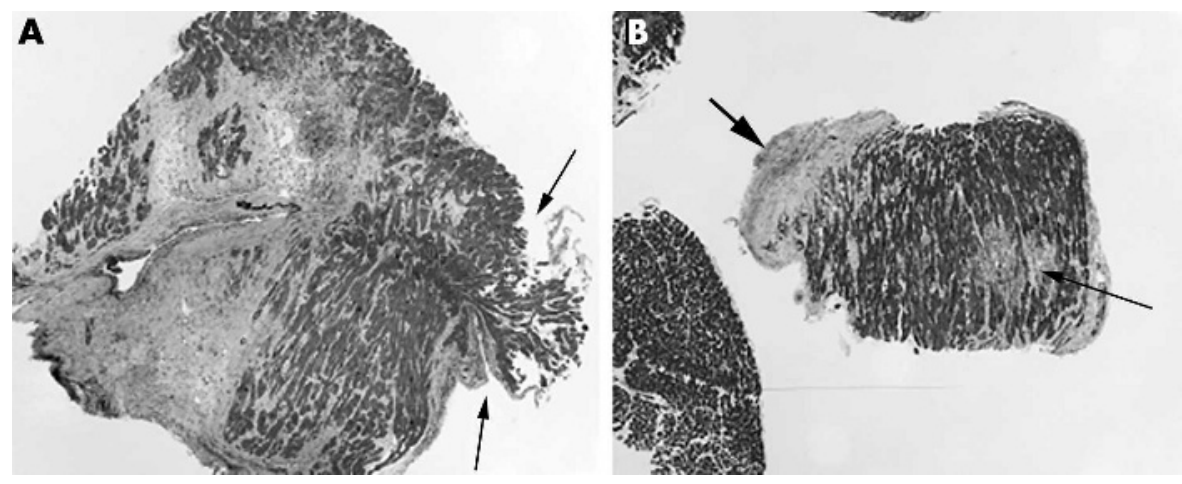

Figure 5 (A) Biopsy artefact showing pinching of the sample at the time of procurement (arrows). Also note the region of fibrosis, which probably represents the site of an earlier biopsy (elastic trichrome stain; original magnification, $\times 2.5$ ). (B) Tissue fragment from a very recent transplant showing endocardial fibrosis, probably secondary to a healed biopsy site (thick arrow). Also note the region of interstitial fibrosis and mixed inflammatory infiltrate consistent with a "vasopressor effect" and probably not the site of a healed episode of rejection (elastic trichrome stain; original magnification, $\times 1.6)$.

\section{Myocardium}

Normal myocardium is made up of approximately one third cardiomyocytes, with the remainder being interstitial cells and vascular endothelium. The myocardium is composed of cardiomyocytes joined at intercalated disks. There is a centrally placed nucleus (or occasionally two nuclei), often with perinuclear lipofuscin. Muscle fibres may run in different directions; however, frank myofibre disarray per se is not common in the normal heart, except at the ventricular apex and junction between the free wall and septum. ${ }^{32}$

Myocyte hypertrophy is generally a consequence of a volume or pressure overloaded ventricle, or a manifestation of a primary cardiomyopathy. Cellular hypertrophy can be the consequence of increased myofibril production or the presence of intracytoplasmic accumulations as a result of metabolic storage disorders, such as Fabry's disease. ${ }^{37}$ Hypertrophy may be physiological or pathological and is a non-specific finding, requiring concomitant clinico-pathological findings for relevance. Myocyte hypertrophy may be evaluated more accurately from nuclear changes as opposed to myocyte dimensions, which may be affected by ventricular dilation and remodelling. Nuclear polyploidy is often seen in hypertrophied cardiomyocytes.

Myocardial disease may also arise from myofibril loss causing atrophy or increased vacuolisation of myocytes, a condition termed myocytolysis. Myofibre loss can also be seen in conjunction with replacement fibrosis, as a consequence of excess physiological stress, ischaemia, or a direct toxic insult. Both myocytolysis and myocyte hypertrophy are very common in areas adjacent to fibrosis. It is possible to confuse normal Purkinje cells with pathological myocytolysis. This pitfall is especially important in left ventricular biopsies. Vacuolisation of the myocytes as a result of contraction band artefact is another common confusing change.

Myofibre disarray often reflects primary myocardial disease and is classically associated with hypertrophic cardiomyopathy. Myofibre disarray may be characterised histologically as cardiomyocytes joining in a disordered fashion, often end to side or myofibres resembling a "basket

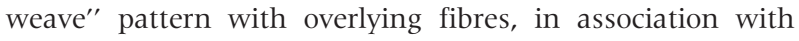
considerable interstitial fibrosis. Myocytes may intersect at various angles, giving the tissue a whorled appearance. Myofibrils may even be seen within the myocyte, running in different directions. ${ }^{23}$ Myofibre disarray is common in healed biopsy sites, such as those in the transplanted heart.

\section{Interstitium}

The interstitium is composed of both structural and adhesive proteins and proteoglycans. The cellular constituents include fibroblasts, histiocytes, smooth muscle cells, myofibroblasts, mast cells, adipocytes (more in the right ventricle), and leucocytes, in addition to a dense capillary network of endothelial cells. It is important to note that the composition of the interstitium changes slightly with more adipocytes and fibrosis (fig 7). ${ }^{32}$ Age related deposition of amyloid is common. $^{38}$

Examination of the interstitium provides information about cellular infiltrates (generally immune/inflammatory) and the consequences of their activity. Lymphocytes may be associated with direct myocyte toxicity, as in acute transplant rejection or myocarditis, or their presence may be entirely benign, as with Quilty lesions. The nature of the inflammatory infiltrate is important to consider-for example, if it includes neutrophils or histiocytes it may be indicative of a more acute or granulomatous process. The inflammatory cell composition may reflect specific conditions and should be correlated with the available clinical history. Interstitial oedema, as demonstrated by a widened interstitium with possibly a subtle pink fibrinous exudate, may suggest an ongoing inflammatory process. Caution should be exercised in identifying interstitial oedema because traction artefact from biopsy procurement can simulate oedema.
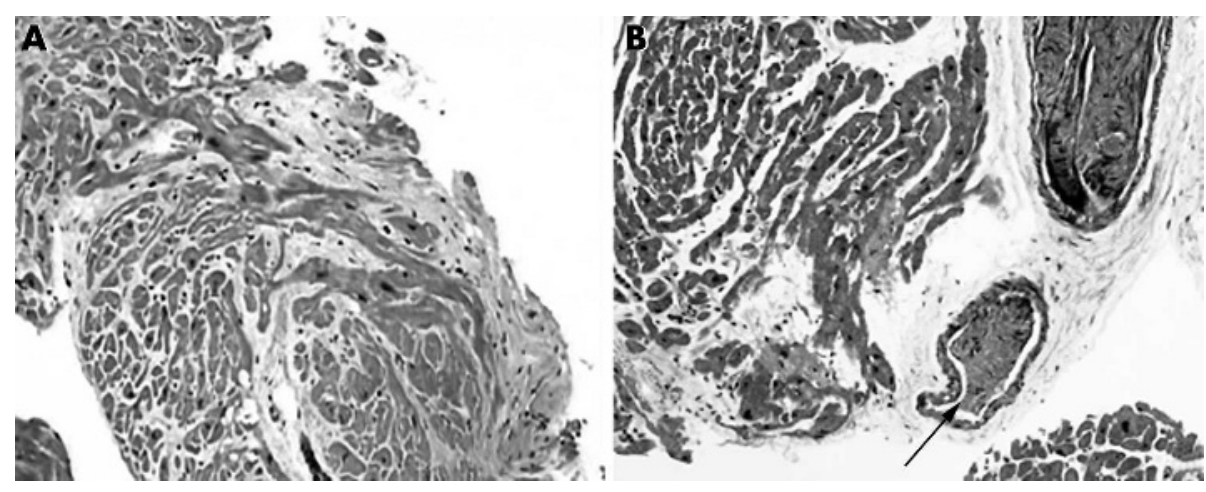

Figure 6 (A) Section showing the typical pattern of myofibre disarray that can be seen at previous biopsy sites (haematoxylin and eosin staining: original magnification, $\times 20$ ). (B) Biopsy artefact showing intussusception of an intramural vessel, not evidence of occlusion/vasculitis (arrow) (elastic trichrome stain; original magnification, $\times 10)$. 

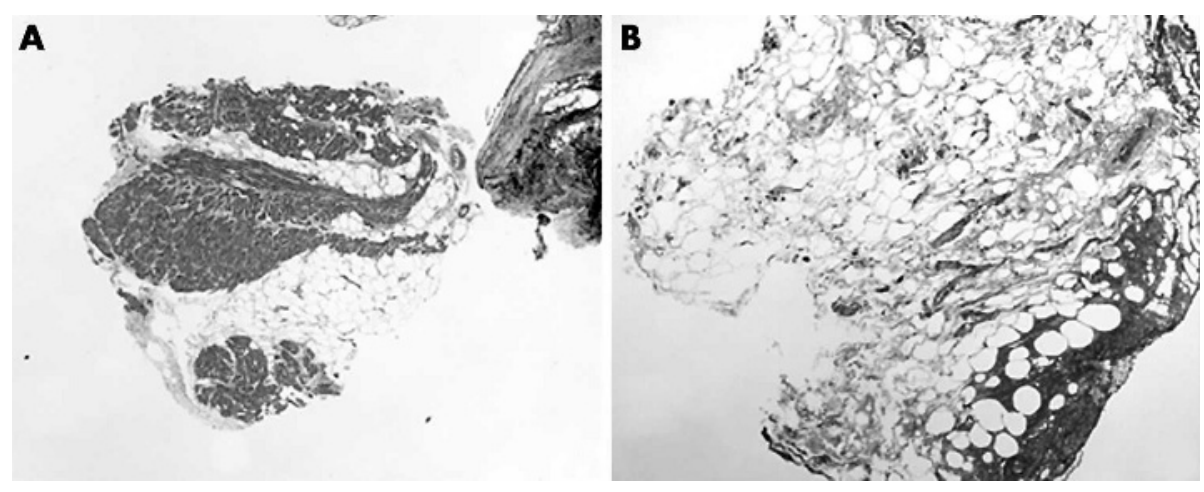

Figure 7 (A) Elastic trichrome stained section showing considerable fat infiltration into the myocardium, near the ventricular apex (original magnification, $\times 2.5$ ). (B) Section showing what appears to be epicardial fat and haemorrhage, suggestive of ventricular perforation. The definitive identification of ventricular perforation would require the evaluation of more sections and the detection of epicardial mesothelium. Prompt notification of such findings should be made to the interventional cardiologist who performed the biopsy (elastic trichrome stain; original magnification, $\times 5$ ).

Immunophenotyping is available to characterise lymphocytic infiltrates if necessary. ${ }^{39-41}$ The presence of lymphocytic atypia should raise the differential diagnosis of lymphoma or post transplant lymphoproliferative disorder in the setting of an allograft heart. ${ }^{42}$ The presence of mast cells or eosinophils should prompt an evaluation for parasitic infection, hypersensitivity reactions (especially drugs), and mastocytosis or hypereosinophilic syndrome-Loeffler's endomyocarditis. The cardiac pathologist should not only facilitate the diagnosis of the heart disease in question, but should also raise the possibility of systemic processes and suggest further investigations that may be warranted.

\section{"Lymphocytes may be associated with direct myocyte toxicity, as in acute transplant rejection or myocarditis, or their presence may be entirely benign, as with Quilty lesions"}

Many of the same aetiologies already mentioned that predispose to endocardial fibrosis also hold true for interstitial fibrosis; however, myocardial changes-such as evidence of coronary artery disease, identification of microinfarcts, microvascular changes, and the presence of cellular infiltrates-are more likely to be present in interstitial fibrosis. In the allograft

\section{Take home messages}

- Although non-invasive imaging techniques have been investigated for the diagnosis of many primary and secondary cardiac conditions, there are still considerable limitations to these techniques, and the endomyocardial biopsy (EMB) remains the gold standard mode of investigation

- The EMB is used widely as a means to follow allograft rejection after heart transplantation, and is also used to diagnose conditions affecting the heart including cardiomyopathies, myocarditis, infiltrative lesions, arrhythmias, and drug toxicities

- $E M B$ is also used as a research tool to investigate the natural history of disease and the cardiotoxicity of new medications

- The EMB procedure is a safe, simple, and effective interventional procedure with a very low rate of morbidity and mortality

- The interpretation of EMB specimens requires knowledge of the patient's clinical history and an appropriate understanding of cardiovascular pathophysiology heart, focal interstitial fibrosis should also be considered in the setting of healed foci of rejection and post transplant graft procurement injury. Certainly, any cellular or interstitial changes seen very shortly after transplantation should be tempered with knowledge of the ischaemic time of the donor heart, donor condition, and subsequent exposure to vasopressors. ${ }^{22}$ The "catecholamine effect" caused by high concentrations of vasopressors before transplantation is manifested as small foci of myocyte damage surrounded by a mixed inflammatory infiltrate that includes neutrophils (fig 5).22 These sites may be mistaken for early evidence of rejection seen within the first few weeks after transplantation. Once healed, they may resemble regions of healed rejection.

\section{Intramural vessels}

Intramural myocardial microvasculature is known to be resistant to atherosclerosis. The most frequent change most pathologists will see in an EMB specimen is allograft vasculopathy (graft vascular disease), which affects epicardial and intramural vessels in donor hearts. The aetiology of this process is uncertain; however, it appears to be the result of a combination of immunological factors and nonimmunological factors related to the transplanted heart/ recipient or may be a side effect of the immunosuppressive drugs. Cardiac allograft vasculopathy appears to affect 30$60 \%$ of individuals within five years of transplantation. ${ }^{43}$ Allograft vasculopathy differs from atherosclerosis in that it is characterised by concentric rather than eccentric intimal hyperplasia, and diffuse lesions affecting both proximal arteries/arterioles with intact internal elastic membranes.

Hypertrophic cardiomyopathy may also have distinctive small vessel changes. In addition to myocyte disarray, wall thickening, and fibrosis, microvascular dysfunction is a common feature of hypertrophic cardiomyopathy, and may be morphologically characterised by reduced arteriolar density and intimal and medial hyperplasia, with increased collagenous and smooth muscle cell components. ${ }^{44}$ Interestingly, the intramural vessel changes seem to occur more frequently in regions with more interstitial fibrosis. ${ }^{45}$

Small vessel thickening may also be seen in diabetes mellitus, systemic arterial hypertension, and in numerous collagen vascular disorders. Amyloidosis may also involve the intramural vessels and cause ischaemia. ${ }^{46}$ Angiography may be normal in individuals with unexplained chest pain for whom hypertrophic cardiomyopathy has not been clinically diagnosed, and the EMB may provide a valuable explanation for this clinical problem. ${ }^{47}$ Microvascular thickening has also been reported in patients with mitral valve prolapse syndrome and in those with atypical chest pain-syndrome $\mathrm{X}^{48}$ The presence of pre-existing coronary artery disease in the donor organ may even be associated with small vessel changes downstream of stenotic epicardial vessels. ${ }^{49}$ 


\section{Adequate specimen}

4 or more good pieces of endomyocardium

Assess all sections

\section{(1) Endocardium}

Normal

$\uparrow$ Thickness

Subendocardial

Fat/fibrosis/inflammation

\section{(2) Myocardium}

Muscle fibre number

Size

Damage

\section{(3) Interstitium}

Normal

Fibrosis

Fat

Oedema

Inflammation

If so - type

\section{(4) Intramural vessels}

\author{
Increased \\ Inflammation \\ Damage
}

Luminal stenosis

\section{(5) Also assess}

Any epicardium
Mesothelial cells

Thrombus

Chordae tendineae

\section{Inadequate specimen}

Tissue is primarily:

\section{Scar}

Fat

Thrombus

2 or fewer pieces of endocardium

\section{Borderline specimen}

3 pieces of endomyocardium

Diagnosis is borderline adequate (see comment)

Comment: mention histological findings - if definite diagnostic features seen then give diagnosis

(such as, myocarditis/amyloid)

Figure 8 The evaluation of an endomyocardial biopsy.

\section{CONCLUSIONS}

The EMB remains the gold standard to assess and diagnose myocardial disease in the living patient (fig 8). Although there have been numerous studies comparing non-invasive imaging techniques to EMB for the diagnosis of graft rejection, infiltrative disorders, and even intracardiac masses, there are still considerable limitations to these techniques. ${ }^{50-52}$ The EMB procedure is a safe, simple, and effective interventional procedure with a very low rate of morbidity and mortality. The interpretation of EMB specimens requires knowledge of the patient's clinical history and an appropriate understanding of cardiovascular pathophysiology.

\section{Authors' affiliations}

K S Cunningham, J Butany, Department of Pathology, University Health Network, University of Toronto, Toronto, Ontario M5G 2CA, Canada J P Veinot, Department of Laboratory Medicine, The Ottawa Hospital, University of Ottawa, Ottawa K1Y 4E9 Canada

\section{REFERENCES}

1 Weinberg M, Fell EH, Lynfield J. Diagnostic biopsy of the pericardium and myocardium. AMA Arch Surg 1958;76:825-9.

2 Sutton DC, Sutton GC. Needle biopsy of the human ventricular myocardium: review of 54 consecutive cases. Am Heart J 1960;60:364-70.

3 Sakakibara S, Konno S. Endomyocardial biopsy. Jpn Heart J 1962;3:537-43.

4 Bulloch RT, Murphy ML, Pearce MB. Intracardiac needle biopsy of the ventricular septum. Am J Cardiol 1965;16:227-33.

5 Richardson PJ. Technique of endomyocardial biopsy-including a description of a new form of endomyocardial bioptome. Postgrad Med J 1975;51:282-5.

6 Brooksby IA, Jenkins BS, Davies MJ, et al. Left ventricular endomyocardial biopsy. I: description and evaluation of the technique, Cathet Cardiovasc Diagn 1977;3:115-21.

7 Caves PK, Stinson EB, Graham AF, et al. Percutaneous transvenous endomyocardial biopsy. JAMA 1973;225:288-91.

8 Ocel JJ, Edwards WD, Tazelaar HD, et al. Heart and liver disease in 32 patients undergoing biopsy of both organs, with implications for heart or liver transplantation. Mayo Clin Proc 2004;79:492-501.

9 Mattos BP, Zettler CG, Pinotti AF, et al. Left ventricular function and endomyocardial biopsy in early and advanced dilated cardiomyopathy. Int J Cardiol 1998;63:141-9.

10 Angelini A, Crosato M, Boffa GM, et al. Active versus borderline myocarditis: clinicopathological correlates and prognostic implications. Heart 2002:87:210-15.

11 Seth S, Thatai D, Sharma S, et al. Clinico-pathological evaluation of restrictive cardiomyopathy (endomyocardial fibrosis and idiopathic restrictive cardiomyopathy) in India. Eur J Heart Fail 2004;6:723-9.

12 Giordano SH, Booser DJ, Murray JL, et al. A detailed evaluation of cardiac toxicity: a phase II study of doxorubicin and one- or three-hour-infusion paclitaxel in patients with metastatic breast cancer. Clin Cancer Res 2002;8:3360-8.

13 D'Amati G, Factor SM. Endomyocardial biopsy findings in patients with ventricular arrhythmias of unknown origin. Cardiovasc Pathol 1996;5:139-44.

14 Amory J, Chou TM, Redberg RF, et al. Diagnosis of primary cardiac leiomyosarcoma by endomyocardial biopsy. Cardiovasc Pathol 1996:5:113-17.

15 Suter TM, Cook-Bruns N, Barton C. Cardiotoxicity associated with trastuzumab (Herceptin) therapy in the treatment of metastatic breast cancer. Breast 2004; 13:173-83.

16 Nieminen MS, Ramo MP, Viitasalo M, et al. Serious cardiovascular side effects of large doses of anabolic steroids in weight liffers. Eur Heart J 1996;17:1576-83.

17 Lombardo T, Tamburino C, Bartoloni G, et al. Cardiac iron overload in thalassemic patients: an endomyocardial biopsy study. Ann Hematol 1995:71:135-41.

18 Barbaro G, Di Lorenzo G, Grisorio B, et al. Incidence of dilated cardiomyopathy and detection of HIV in myocardial cells of HIV-positive patients. Gruppo Italiano per lo Studio Cardiologico dei Pazienti Affetti da AIDS. N Engl J Med 1998:339:1093-9.

19 Drury JH, Labovitz AJ, Miller LW. Echocardiographic guidance for endomyocardial biopsy. Echocardiography 1997;14:469-74.

20 Takemura G, Fujiwara H, Horike K, et al. Ventricular expression of atrial natriuretic polypeptide and its relations with hemodynamics and histology in dilated human hearts. Immunohistochemical study of the endomyocardial biopsy specimens. Circulation 1989;80:1137-47.

21 Frustaci A, Chimenti C, Bellocci F, et al. Histological substrate of atrial biopsies in patients with lone atrial fibrillation. Circulation 1997;96:1 180-4.

22 Billingham ME. Pathology of heart transplantation. In: Solez K, Racusen LC, Billingham ME, eds. Solid organ transplant rejection: mechanisms, pathology and diagnosis. New York: Marcel Dekker, Inc, 1996:137-59.

23 Winters GL, Schoen FJ. Pathology of cardiac transplantation. In: Silver MD, Gotlieb Al, Schoen FJ, eds. Cardiovascular pathology. Philadelphia: Churchill Livingstone, 2001:725-59.

24 Zerbe TR, Arena V. Diagnostic reliability of endomyocardial biopsy for assessment of cardiac allograft rejection. Hum Pathol 1988;19:1307-14.

25 Pauschinger M, Chandrasekharan K, Noutsias $M$, et al. Viral heart disease: molecular diagnosis, clinical prognosis, and treatment strategies. Med Microbiol Immunol (Berl) 2004;193:65-9.

26 Crotty TB, Li CY, Edwards WD, et al. Amyloidosis and endomyocardial biopsy: correlation of extent and pattern of deposition with amyloid immunophenotype in 100 cases. Cardiovasc Pathol 1995;4:39-42.

27 de Souza MM, Franco M, Almeida DR, et al. Comparative histopathology of endomyocardial biopsies in chagasic and non-chagasic heart transplant recipients. J Heart Lung Transplant 2001;20:534-43.

28 Baan CC, Knoop CJ, van Gelder T, et al. Contribution of the inflammatory response to cardiac allograft rejection: histopathologic analysis of serial endomyocardial biopsies. Transplant Proc 1998;30:1104-6.

29 Duong Van Huyen JP, Fornes P, Guillemain R, et al. Acute vascular humoral rejection in a sensitized cardiac graft recipient: diagnostic value of $C 4 d$ immunofluorescence. Hum Pathol 2004;35:385-8. 
30 Fishbein MC, Kobashigawa J. Biopsy-negative cardiac transplant rejection: etiology, diagnosis, and therapy. Curr Opin Cardiol 2004;19:166-9.

31 Veinot JP. Diagnostic endomyocardial biopsy pathology-general biopsy considerations, and its use for myocarditis and cardiomyopathy: a review. Can J Cardiol 2002; 18:55-65.

32 Veinot JP, Ghadially FN, Walley VM. Light microscopy and ultrastructure of the blood vessels and heart. In: Silver MD, Gotlieb Al, Schoen FJ, eds. Cardiovascular pathology. Philadelphia: Churchill Livingstone, 2001:30-53.

33 Cunningham K, Davies RA, Catching J, et al. Pathologic quiz case: a young woman with eosinophilia and heart failure. Primary hypereosinophilic syndrome with Loeffler endocarditis. Arch Pathol Lab Med 2005; 129:e29-30.

34 Angelov A, Kulova A, Gurdevsky M. Endocardial fibroelastosis. Clinicopathological study of 38 cases. Pathol Res Pract 1984;178:384-8.

35 Gopal S, Narasimhan U, Day JD, et al. The Quilty lesion enigma: focal apoptosis/necrosis and lymphocyte subsets in human cardiac allografts. Pathol Int 1998;48:191-8.

36 Barone JH, Fishbein MC, Czer LS, et al. Absence of endocardial lymphoid infiltrates (Quilty lesions) in nonheart transplant recipients treated with cyclosporine. J Heart Lung Transplant 1997;16:600-3.

37 Koitabashi N, Utsugi T, Seki R, et al. Biopsy-proven cardiomyopathy in heterozygous Fabry's disease. Jpn Circ J 1999;63:572-5.

38 Kyle RA, Spittell PC, Gertz MA, et al. The premortem recognition of systemic senile amyloidosis with cardiac involvement. Am J Med 1996;101:395-400.

39 Groeneveld K, Balk AH, Ouwehand AJ, et al. Phenotype of endomyocardial biopsy-derived T-lymphocyte cultures and chronic rejection after heart transplantation. Transpl Int 1992;5(suppl 1):S228-30.

40 van Besouw NM, Balk AH, Mochtar B, et al. Phenotypic analysis of lymphocytes infiltrating human cardiac allografts during acute rejection and the development of graft vascular disease. Transpl Int 1996;9/suppl 1):S234-6

41 Trentin L, Zambello R, Faggian G, et al. Phenotypic and functional characterization of cytotoxic cells derived from endomyocardial biopsies in human cardiac allografts. Cell Immunol 1992;141:332-41.
42 Kelley MP, Narula N, Loh E, et al. Early post-transplant lymphoproliferative disease following heart transplantation in the absence of lymphocytolytic induction therapy. J Heart Lung Transplant 2000; 19:805-9.

43 Valantine H. Cardiac allograft vasculopathy after heart transplantation: risk factors and management. J Heart Lung Transplant 2004;23(suppl 5):S187-93

44 Cecchi F, Olivotto I, Gistri R, et al. Coronary microvascular dysfunction and prognosis in hypertrophic cardiomyopathy. N Engl J Med 2003:349:1027-35.

45 Maron BJ, Wolfson JK, Epstein SE, et al. Intramural ("small vessel") coronary artery disease in hypertrophic cardiomyopathy. J Am Coll Cardiol 1986;8:545-57.

46 Yamano S, Motomiya K, Akai Y, et al. Primary systemic amyloidosis presenting as angina pectoris due to intramyocardial coronary artery involvement: a case report. Heart Vessels 2002;16:157-60.

47 Yamamoto S, James TN, Kawamura K, et al. Cardiocytic apoptosis and capillary endothelial swelling as morphological evidence of myocardial ischemia in ventricular biopsies from patients with angina and normal coronary arteriograms. Coron Artery Dis 2002;13:25-35.

48 Osamichi S, Kouji K, Yoshimaro I, et al. Myocardial glucose metabolism assessed by positron emission tomography and the histopathologic findings of microvessels in syndrome X. Circ J 2004;68:220-6.

49 Hong H, Aksenov S, Guan X, et al. Remodeling of small intramyocardial coronary arteries distal to a severe epicardial coronary artery stenosis. Arterioscler Thromb Vasc Biol 2002;22:2059-65.

50 Schoels M, Dengler TJ, Richter R, et al. Detection of cardiac allograft rejection by real-time PCR analysis of circulating mononuclear cells. Clin Transplant 2004; 18:513-17.

51 Bomma C, Rutberg J, Tandri H, et al. Misdiagnosis of arrhythmogenic right ventricular dysplasia/cardiomyopathy. J Cardiovasc Electrophysiol 2004; 15:300-6.

52 Gowda RM, Khan IA. Clinical perspectives of primary cardiac lymphoma. Angiology 2003;54:599-604, .

\section{bmjupdates+}

bmjupdates+ is a unique and free alerting service, designed to keep you up to date with the medical literature that is truly important to your practice.

bmjupdates+ will alert you to important new research and will provide you with the best new evidence concerning important advances in health care, tailored to your medical interests and time demands.

Where does the information come from?

bmjupdates+ applies an expert critical appraisal filter to over 100 top medical journals A panel of over 2000 physicians find the few 'must read' studies for each area of clinical interest

Sign up to receive your tailored email alerts, searching access and more...

www.bmjupdates.com 